\title{
Morphognostic Analysis of Fetal Ilium for Sex Determination
}

Luv $\mathbf{S}^{2}$, Aarti $\mathbf{R}^{1}$, Kamal $\mathbf{S}^{1^{*}}$ and Tarun $\mathbf{D}^{2}$

${ }^{1}$ Department of Anatomy, Pt. B. D. Sharma Post Graduate Institute of Medical Sciences, University of Health Sciences, Rohtak, Haryana, India

${ }^{2}$ Department of Forensic Medicine, Pt. B. D. Sharma Post Graduate Institute of Medical Sciences, University of Health Sciences, Rohtak, Haryana, India

"Corresponding author: Kamal S, Department of Anatomy, Pt. B. D. Sharma Post Graduate Institute of Medical Sciences, University of Health Sciences, Rohtak, Haryana, India, Tel: 919812531007; E-mail: drkamalanatomy@gmail.com

Received date: August 23, 2017; Accepted date: September 04, 2017; Published date: September 08, 2017

Copyright: () 2017 Luv S, et al. This is an open-access article distributed under the terms of the Creative Commons Attribution License, which permits unrestricted use, distribution, and reproduction in any medium, provided the original author and source are credited.

\begin{abstract}
Sexing of an individual by skeletal remains can be well established by using pelvis in adults especially on fused bones only. Forensic fetal Osteology is the field which needs to be explored for sex determination. The 34 pairs of Fetal pelvis bones (>22 weeks and $<22$ weeks of gestation) in different stages of decomposition were studied for morphognostic parameters i.e. Genove's composite arch and Schutkowski's Arch Criterion, Sciatic Notch Angle and Auricular Surface Elevation and compared with 26 pairs (10 Males+16 Females) of adult hip bones with already known sex. Criteria evaluated and their results were good enough for establishing sex in fetal specimens especially of $>22$ weeks with $100 \%$ accuracy. The findings in our study showed $100 \%$ accuracy in determination of sex by fetal ilium bone which can be used as a potential tool for sex determination.
\end{abstract}

Keywords: Sex; Fetal; Ilium; Arch

\section{Introduction}

Establishment of sexual dimorphism is most contested topic of research for forensic pathologists. Multiple researchers have tried to establish sex from skeletal remains. Even sexual dimorphism have been establishes in smallest bones of the body i.e. ear ossicles [1-3]. Pelvis is the part of skeleton in which maximum skeletal dimorphism has been established with considerable investigation in the greater sciatic notch and auricular surface area [4]. But these methods are focussed on adults especially on fused bones. Forensic fetal Osteology is the field which needs to be explored for sex determination. Multiple researchers from all over the world has tried to establish sexual dimorphism in fetal collections by visual methods and metric analysis and it has been held that dimorphism may exist from an early age [5-8]. But it is not sufficient enough until the skeletal maturation has taken place under the influence of puberty. The concept of composite arch was proposed by Genoves and it has been successfully utilised in determination of sex with $80 \%$ accuracy for males and $88 \%$ for females [9]. Similarly, the arch criterion was introduced by Schutkowski in sub-adult skeletal specimens with a correct sex allocation accuracy of $81.5 \%$ for males and $60 \%$ for females, which was very similar to composite arch criterion. Schutkowski's method for sex determination allows rapid evaluation of morphological features but the scoring procedure is not precisely defined, making the evaluation process overly subjective and dependent on investigator's experience leading to inconsistent evaluation of morphological features [10]. Vlak D has argued the Schutkowski's morphological method did not predict sex accurately in all populations [11].

Sciatic Notch angle and Auricular surface elevation has also been assessed by multiple researchers with contradictory results on visual photographic and metric assessments Holocomb [12]. Sciatic notch has received the most attention as in adults sexual difference are well established and is easily seen in fetal skeletal remains but unfortunately has been proved to provide ambiguous results. Auricular Surface has been assessed by Weaver (1980), Hunt (1990), Mittler and Sheridan (1992) and Sutter in 2003. Variable results has proved it to be an inconsistent trait to classify fetal pelvic bones as male or female [13-16]. HFV Cardaso suggested that because of great variations in morphology, difficulty in interpreting criteria, careful examination of sex related morphology in fetal skeletal remains needs to be investigated [17]. The initial goal of the study was to test the accuracy of the above mentioned two traits (composite arch and arch criterion) for sex determination of fetal ilia along with sexual dimorphism in sciatic notch angle (both morphologically and morphometrically) and asymmetry of auricular surface. But it was also extended to the adult hip bones with known sex for the applicability of these criteria for sex determination and comparison of results with fetal ilium bones.

\section{Material and Methods}

The 34 pairs of Fetal pelvis bones (22 Males+12 Females) in different stages of decomposition were studied and compared with 26 pairs (10 Males+16 Females) of adult hip bones with already known sex. The adult hip bones of known sex were obtained at random from the skeletal collection of Department of Anatomy at Pt. B. D. Sharma PGIMS, Rohtak.

\section{Collection of fetal bones}

Bilateral fetal pelvic bones were collected from 34 unidentified dead fetuses age ranging from 16 weeks to 38 weeks that were brought in the department of Forensic medicine for the postmortem examination. Only unidentified and unclaimed bodies were used for sample collection ruling out the ethical issues.

\section{Inclusion/exclusion criteria}

Only intact fetal pelvic bone sample from recognizable sex were included. Fragmentary and unidentifiable fetal remains and individuals with any skeletal defects, congenital anomalies and fragmentary remains were excluded from the study. The age of the 
subjects was derived from established bone age ossification centre based parameters and crown rump length of the fetus which was measured in supine position of the fetus by using infantometer.

\section{Method of Dissection}

The pelvic bones were removed causing minimal disfigurements of the body by making a horizontal incision over anterior aspect of pelvic cavity and separated from adjoining soft tissues.

\section{Preparation of the sample for study}

The separated bones from the pelvis were kept in a jar containing plain tap water and the jars were labeled with the case no, sex and approximate calculated age of the fetus. No chemical was used to clean the bones to avoid any erosion of the bones. The bones were examined intermittently and once the soft tissue got separated, the bones were cleaned and dried at room temperature. All the bones were kept separately in duly numbered packets for further analysis.

\section{Measurements for the study}

Fetal bone samples were grouped into two age groups i.e. $<22$ weeks and $>22$ weeks. The age has been taken as a reference point keeping in view that the ossification of fetal pelvic bone starts with ilium which undergoes endochondral ossification at 9.5 weeks and development of trabecular bone is evident from 22 weeks and onwards leading to the remodeling of the bone [18]. Each sample was measured separately and the following measurements were noted after careful examination.

\section{Morphognostic parameters of fetal ilium}

- Composite arch criteria by genoves: Assesses whether the curved line that extends dorsally from the anterior rim of the sciatic notch have the same circumference and overlap each other or do not overlap (Figure 1) [9].

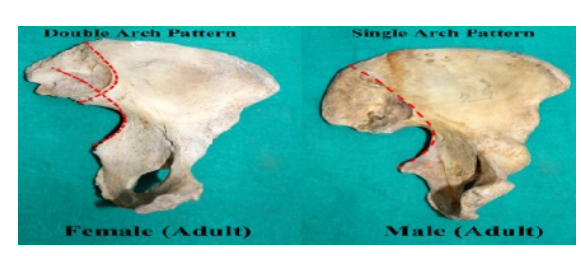

Figure 1: Showing adult hip bone for composite arch criteria by genoves (Double and Single Arch Pattern).

- Arch criterion: The ilium was arranged with the length of the anterior sciatic notch vertically oriented [10]. An arch was drawn, starting from the anterior portion of the notch either crossing through or passing above the auricular surface (Figure 2) [10].

Each ilium was laid on a flat surface and an orthogonal photograph was taken and studied for composite arch and arch criterion. The traits were scored separately in fetal and adult bones.

- Sciatic Notch Angle (0):

1. Morphologically: By visual assessment (Acute/Obtuse)

2. Morphometrically: By measuring the angle with protractor

- Auricular Surface Elevation: Elevation of the auricular surface (F) along anterior and posterior edges denoted a raised surface, while no elevation denoted a not raised surface. Additionally, if only a portion of the auricular surface was raised, the portion of the bone raised was recorded as superior, anterior, inferior and/or posterior raised. The number of sides raised was documented following Sutter's (2003) rank system from 0 to 4 [16]. To analyze the auricular surface each raised side was scored as follows: No Sides Raised=Rank 0,

One Side Raised=Rank 1,

Two Sides Raised=Rank 2,

Three Sides Raised=Rank 3,

Completely Raised=Rank 4 .

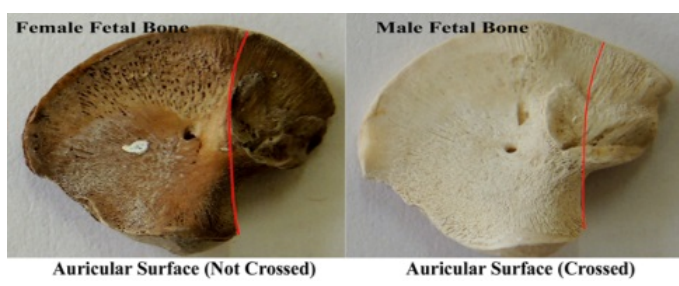

Figure 2: Showing fetal ilium bone for arch crossing and not crossing the auricular surface.

All the measurements were taken separately on fetal ilium bone of each side by three different observers, 1 PG Student, 1 Expert, and 1 Osteologist after standardizing the points to be taken into consideration for measurements. Assessments were repeated by each observer after 3 weeks time in order to observe for intra observer and inter observer errors. Then the results were computed by SPSS software.

\section{Observations and Results}

There were insignificant intra observer and inter observer errors while observing the morphognostic parameters. The results have been observed are as follows (Tables 1 to 5). Single arch pattern was observed in $100 \%$ specimens of female fetal bones of $>22$ weeks age group and in adult male bones respectively. While double arch pattern was observed in $75 \%$ specimens of male fetal bones of $>22$ weeks, $66.6 \%$ specimens of male fetal bones of $<22$ weeks, $100 \%$ specimens of female fetal bones of $<22$ weeks and $100 \%$ in adult female bones. Single arch pattern is indicative of female bones in $>22$ weeks and adult male bones. Double arch pattern is indicative of adult male bones while inconclusive in fetal bones. Arch criterion by Schutkowski showed the similar results as that of Genoves with slight differences. The arch crossed the auricular surface in $75 \%$ and $71.1 \%$ of male fetal bones in $>$ 22 weeks and $<22$ weeks respectively and $100 \%$ in Female fetal bones of $<22$ weeks and adult female bones. Thus, arch criterion is inconclusive in $<22$ weeks for determination of sex. However, the arch borders the auricular surface in $100 \%$ of females fetal bones in $>22$ weeks. Hence it can be used as a tool for determination of sex in $>22$ weeks fetal ilium bones. On comparison of sciatic notch angle in adults morphometrically (Table 3 ), statistically significant difference ( $p$ value $<0.05$ ) was observed while visual assessment showed acute angles in both male and female adult hip bones. While fetal bones of all the age groups, in both sexes revealed obtuse sciatic notch angle on visual assessment and insignificant difference on morphometric assessment ( $\mathrm{p}$-value $>0.05$ ). While observing the Auricular surface elevation 
Citation: Luv S, Aarti R, Kamal S and Tarun D (2017) Morphognostic Analysis of Fetal llium for Sex Determination. J Forensic Res 8: 389. doi:

Page 3 of 5

according to Sutter Ranking System, Rank (0) was observed in $50 \%$ male fetal bones in $>6$-month age group and in $42.8 \%$ in $<6$ month age group and $33.3 \%$ in $<6$ month age group of females. Rank (1) was observed only in $14.2 \%$ male fetal bones in $<6$-month age group. Rank
(2) was observed more in females i.e. $66.6 \%$ in $<6$-month age group and in $33.3 \%$ in $>6$ month age group. Rank (3) was observed in $66.6 \%$ of females in $>6$-month age group. In adults, $80 \%$ of male bones were in rank (2) and $85.8 \%$ of female bones were in Rank (3).

\begin{tabular}{|c|c|c|c|c|c|c|}
\hline \multirow[t]{3}{*}{ Arch Pattern } & \multicolumn{4}{|c|}{ Fetal Bone } & \multicolumn{2}{|c|}{ Adult Bone } \\
\hline & \multicolumn{2}{|c|}{ Male } & \multicolumn{2}{|c|}{ Female } & \multirow[t]{2}{*}{ Male } & \multirow[t]{2}{*}{ Female } \\
\hline & $>22$ Weeks & $<22$ Weeks & $>22$ Weeks & $<22$ Weeks & & \\
\hline Double & $75 \%$ & $66.60 \%$ & $0 \%$ & $100 \%$ & $0 \%$ & $100 \%$ \\
\hline Single & $25 \%$ & $33.30 \%$ & $100 \%$ & $0 \%$ & $100 \%$ & $0 \%$ \\
\hline
\end{tabular}

Table 1: Composite arch criteria by genoves.

\begin{tabular}{|c|c|c|c|c|c|c|}
\hline \multirow{3}{*}{$\begin{array}{c}\text { Auricular Surface } \\
\text { (Crossed/Not Crossed) }\end{array}$} & \multicolumn{4}{|c|}{ Fetal Bone } & \multicolumn{2}{|c|}{ Adult Bone } \\
\hline & \multicolumn{2}{|c|}{ Male } & \multicolumn{2}{|c|}{ Female } & \multirow[b]{2}{*}{ Male } & \multirow[b]{2}{*}{ Female } \\
\hline & $>22$ Weeks & $<22$ Weeks & $>22$ Weeks & $<22$ Weeks & & \\
\hline Crossed & $75 \%$ & $71.70 \%$ & $0 \%$ & $100 \%$ & $0 \%$ & $100 \%$ \\
\hline Not Crossed & $25 \%$ & $28.30 \%$ & $100 \%$ & $0 \%$ & $100 \%$ & $0 \%$ \\
\hline
\end{tabular}

Table 2: Arch criterion.

\begin{tabular}{|l|l|l|}
\hline Sciatic Notch Angle (0) & Male & Female \\
\hline Visually Assessed & Acute & Acute \\
\hline Morphometrically Measured & $52.50 \pm 2.5$ & -- \\
\hline
\end{tabular}

Table 3: Sciatic notch angle (0) in adult bones.

\begin{tabular}{|c|c|c|c|c|c|c|}
\hline \multirow{2}{*}{ Sciatic Notch Angle (0) } & \multicolumn{3}{|c|}{$<22$ Weeks } & \multicolumn{3}{|c|}{$>22$ Weeks } \\
\hline & Male & Female & p-value & Male & Female & p-value \\
\hline Visually Assessed & Obtuse & Obtuse & - & Obtuse & Obtuse & - \\
\hline Morphometrically Measured & $1230 \pm 9.91$ & $1210 \pm 5.46$ & $>0.05$ & $1200 \pm 5.58$ & $1210 \pm 4.50$ & $>0.05$ \\
\hline
\end{tabular}

Table 4: Sciatic notch angle (0) in fetal bone.

\begin{tabular}{|c|c|c|c|c|c|c|c|}
\hline \multirow{2}{*}{\multicolumn{2}{|c|}{ Auricular Surface Elevation }} & \multicolumn{4}{|c|}{ Fetal Bone } & \multicolumn{2}{|c|}{ Adult Bone } \\
\hline & & \multicolumn{2}{|c|}{ Male } & \multicolumn{2}{|c|}{ Female } & \multirow[b]{2}{*}{ Male } & \multirow[b]{2}{*}{ Female } \\
\hline Rank & Raised Sides & $>22$ Weeks & $<22$ Weeks & $>22$ Weeks & $<22$ Weeks & & \\
\hline 0 & No Sides Raised & $50 \%$ & $42.80 \%$ & $0 \%$ & $33.30 \%$ & $0 \%$ & $0 \%$ \\
\hline-1 & One Side Raised & $0 \%$ & $14.20 \%$ & $0 \%$ & $0 \%$ & $0 \%$ & $0 \%$ \\
\hline-2 & Two Sides Raised & $25 \%$ & $42.80 \%$ & $33.30 \%$ & $66.60 \%$ & $80 \%$ & $14.20 \%$ \\
\hline-3 & Three Sides Raised & $25 \%$ & $0 \%$ & $66.60 \%$ & $0 \%$ & $20 \%$ & $85.80 \%$ \\
\hline-4 & Completely Raised & $0 \%$ & $0 \%$ & $0 \%$ & $0 \%$ & $0 \%$ & $0 \%$ \\
\hline
\end{tabular}

Table 5: Auricular surface elevation. 


\section{Discussion}

There have been multiple studies of the sciatic notch. The qualitative examinations included the symmetry of the sciatic notch shape (Symmetric and asymmetric), an angle evaluation (visual estimate of more than 900), and a sciatic notch depth assessment (shallow and deep). The auricular surface elevation, iliac blade curvature and arch criteria were also studied. One of the earliest studies of the sciatic notch is Thomson's review of the foetal pelvis (1899). In this study, Thomson's states that the sciatic notch was wider and shallower in females than in males, a fact which he found surprisingly. It should be noted that Thomson examined intact pelvis and measured the width of the sciatic notch as the length from the anterior greater sciatic notch to the margin of the sacrum that met the posterior inferior iliac spine Posteriorly. The techniques used in this study does not correspond the standardised measurement currently applied [19].

Schutkowski [10] examined the Spitalfields London Juvenile Skeletal Collection, which contained individuals aged from birth to 11years. The iliac traits evaluated included the greater sciatic notch depth and angle, iliac crest curvature and arch criterion. Schutkowski determine that the sciatic notch was particularly diagnostic of sex. He visually assessed females as having angles more than 900 and males having 900. On the basis of arch criterion, whiles males were correctly identified $95 \%$ of the time, this trait had a fairly low reliability rate of only $70-75 \%$ because while males often presented the male features, female often lacked the definite female trait $[10,20]$. While in the present study in adults both male and female hip bone represents acute angle on visual assessment proving it inconclusive while on morphometric assessment significant differences have been observed. Using an adjusted version of Genove's [9] composite arch method, Schutkowski used an arched criterion method evaluating whether an arch along the anterior border of the sciatic notch either crossed the center or the anterior rim of the auricular surface. Schutkowski determined that $73.3 \%$ of males and $70.6 \%$ of females were correctly sexed with this trait $[9,10]$. However, sample size of 29 males and 22 females has an effect on the overall reliability of the study. Schutkowski's findings were not replicated by Vlak et al. [11] on modern sub-adult Portuguese sample.

Vlak [11] opined that the greater sciatic notch is recognisable in early foetal development, it is usually well preserved in archaeological forensic remains, and results of previous studies have shown a statistically significant level of sexual dimorphism. Although Schutkowski obtained a modest correct sex allocation accuracy (72\%) with the arch criterion, Sutter's test shows an increased performance $(82.3 \%)$, suggesting that the trait is useful for sex determination of subadult skeletal remains $[10,16]$. However, a closer inspection of Sutter's results reveals a puzzling finding, which may result from the intricacy of sex-related morphologies in sub-adult ilia. In his study, Sutter considered "bordering" the associated expression of the female trait and "crosses" the expression of the male trait [16].

However, Schutkowski described the female pattern as when "the arch crosses the auricular surface", and the male pattern as bordering of the arch onto the lateral rim of the auricular surface. The results of the current study are similar to the results proposed by Sutter, i.e. Crossing as male pattern in all the age groups, while bordering as Female pattern in age group of $>22$ weeks. It could have been said as unreliable trait for sex determination of sub-adult ilia but as the results are seen in adult pelvic bones which have been established for clear sexual dimorphism, it may suggest a reliable criteria for determination of sex of fetal ilia in $>22$ weeks age group $[10,16]$.
In males, there is no ridge in the relevant "arc area", although some males show a distinct line which parallels the symphyseal edge, but only at about age 25 years. Mittler and Sheridan using the elevation of the auricular surface for sex determination in sub-adults, also suggest this trait to be age-dependent. In older sub-adults ( $>10$ years), an elevated articular surface was indicative of the female sex with a virtually $100 \%$ probability, although failure to develop a surface elevation was not comparably reliable for males (74.4\%). Similar results have been produced in the present study showing elevated auricular surface as female pattern and No sides raised as Male pattern in fetal bones [15].

For determination of sex these traits have not proven to be $100 \%$ reliable due to problems which arise from the scoring of morphological methods \& subjectivity. Present study has tried to minimize the errors by standardizing the points to be taken into consideration for measurements. Metric methods may prove to be more objective and reliable but pose difficulty in their applicability. Because different approaches are to be followed for sex determination of skeletal remains (adult/fetal), morphological criteria are evaluated and their results are fairly good enough for establishing sex in fetal remains especially in fetal specimens with $100 \%$ accuracy in $>22$ weeks age groups. 22 weeks can be considered as the age demarcating criterion as $100 \%$ accurate sex determination is observed in age group $>22$ weeks while all morphognostic parameters failed to discriminate sex of fetal bones of $<22$ weeks. It may be suggested that trabecular bone formation and remodeling under hormonal influence leads to appearance of sexual dimorphic changes in the bone.

\section{Conclusion}

The findings in our study showed $100 \%$ accuracy in determination of sex by fetal ilium bone in $>22$ weeks of age group which can be used as a potential tool for forensic investigation for evaluation of sex in severely mutilated and decomposed bodies during postmortem examination.

\section{References}

1. Singh K, Chhabra S, Sirohiwal BL, Yadav SPS (2012) Morphometry of malleus a possible tool in sex determination. J Forensic Res.

2. Singh K, Rohilla A, Rohilla J (2016) Incus morphometry: A possible tool in sex determination. J Forensic Res.

3. Singh K, Rohilla A (2017) Sexual dimorphism in stapes by discriminant functional analysis. Int J Res Med Sci 5: 3534-3539.

4. Fehling $\mathrm{H}$ (1876) The shape of the pelvis in the fetus and newborn and its relation to that in the adult. Arch Gyna Obst 10: 1-80.

5. Scheur L, Black S (2000) Developmental Juvenile Osteology: Elsevier Academic Press. Amsterdam, USA.

6. Morton DG (1942) Observations of the development of pelvic conformation. Am J Obstet Gynecol 44:799-819.

7. Reynolds EL (1945) The bony pelvic girdle in early infancy. Am J Phys Anthropol 3:321-354.

8. Phenice TW (1969) A Newly developed method of sexing the os pubis. Am J Phys Anthropol 30: 297-302.

9. Genove SL (1959) Estimation of sexual differences in the coxal bone: Metric differences and morphological differences. Bull Mem Soc Anthropol Paris 10: 3-95.

10. Schutkowski H (1993) Sex determination of infant and juvenile skeletons: I. Morphognostic features. Am J Phys Anthropol 90:199-205.

11. Vlak D, Roksandic M, Schillaci MA (2008) Greater sciatic notch as a sex indicator in juveniles. Am J Phys Anthropol 137: 309-315. 
Citation: Luv S, Aarti R, Kamal S and Tarun D (2017) Morphognostic Analysis of Fetal llium for Sex Determination. J Forensic Res 8: 389. doi: 10.4172/2157-7145.1000389

Page 5 of 5

12. Holcomb SM, Konigsberg LW (1995) Statistical study of sexual dimorphism in the human fetal sciatic notch. Am J Phys Anthropol 97:113-125.

13. Weaver DS (1980) Sex differences in the ilia of a known sex and age sample of fetal and infant skeletons. Am J Phys Anthropol 52:191-195.

14. Hunt DR (1990) Sex determination in the subadult ilia: An indirect test of weaver's nonmetric sexing method. J Forensic Sci 35: 881-885.

15. Mittler DM, Sheridan SG (1992) Sex determination in subadults using auricular surface morphology: A forensic science perspective. J Forensic Sci 37:1068-1075.

16. Sutter RC (2003) Nonmetric subadult skeletal sexing traits: I. A blind test of the accuracy of eight previously proposed methods using prehistoric known-sex mummies from Northern Chile. J Forensic Sci 48:927-935.
17. Cardoso HF, Saunders SR (2008) Two arch criteria of the ilium for sex determination of immature skeletal remains: A test of their accuracy and an assessment of intra- and inter-observer error. Forensic Sci Int 178: 24-29.

18. Cunningham CA, Black SM (2009) Anticipating bipedalism: Trabecular organization in the newborn ilium. J Anat 214: 817-829.

19. Thomson A (1899) The sexual differences of the foetal pelvis. J Anat Physiol 33: 359-380.

20. Schutkowski H (1987) Sex determination of fetal neonate skeletons by means of discriminant analysis. Int J of Anthropol 2: 347-352. 